\title{
Single Molecule as a Local Acoustic Detector for Mechanical Oscillators
}

\author{
Yuxi Tian, ${ }^{\dagger}$ Pedro Navarro, and Michel Orrit ${ }^{*}$ \\ MoNOS, Huygens-Kamerlingh Onnes Laboratory, Universiteit Leiden, 2300 RA Leiden, Netherlands
}

(Received 24 July 2014; published 26 September 2014)

\begin{abstract}
A single molecule can serve as a nanometer-sized detector of acoustic strain. Such a nanomicrophone has the great advantage that it can be placed very close to acoustic signal sources and high sensitivities can be achieved. We demonstrate this scheme by monitoring the fluorescence intensity of a single dibenzoterrylene molecule in an anthracene crystal attached to an oscillating tuning fork. The characterization of the vibration amplitude and of the detection sensitivity is a first step towards detection and control of nanomechanical oscillators through optical detection and feedback.
\end{abstract}

DOI: 10.1103/PhysRevLett.113.135505

PACS numbers: 81.07.Oj, 07.10.Cm, 43.35.Sx, 87.80.Nj

Introduction.-Acoustic vibrations are central to communication in our daily lives, but also form the basis of many technologies such as sonar, seismography, or ultrasound medical imaging. The main advantage of acoustic waves is their faculty to propagate in media which absorb or scatter electromagnetic waves. A major drive to achieving smaller, faster, and more sensitive sound detectors is the perspective of generating, detecting, and controlling sound at nanometer scales, which would open new avenues for acoustic microscopy $[1,2]$, as well as for manipulation and cooling of acoustic degrees of freedom down to the quantum regime [3]. Single gold nanoparticles have been shown to be sensitive vibration detectors in solution [4]. In recent years, thanks to spectacular progress in nanoscience, nanometer-scale oscillators have been applied to accurate mass measurements down to the mass of single atoms, and to bacterium screening $[5,6]$. Nanomechanical oscillators are promising candidates as quantum systems that can be manipulated. The vibration amplitude, phase, and even the quantum state of a nanomechanical oscillator could be read through coupling to a quantum system such as a qubit, an optical cavity, a single-electron transistor, a SQUID or a point contact between two conductors [7-10]. Recently, Puller et al. have put forward the theoretical possibility to detect the displacement and to manipulate the state of a nanomechanical oscillator through the optical fluorescence signal of a single molecule [11]. The aim of the present Letter is to demonstrate such detection experimentally, and to provide measurements of the sensitivity in a wellcontrolled and well-understood case. The oscillator will be a quartz crystal tuning fork, causing mechanical deformations of the host crystal around the molecule under optical study.

To selectively detect individual molecules, the molecules have to be separated from each other $[12,13]$. At cryogenic temperature and in suitable rigid matrixes, the absorption spectrum of a molecule presents an extremely narrow electronic transition that occurs without any creation or annihilation of phonons, and is therefore called the zerophonon line (ZPL). Its linewidth is chiefly determined by the lifetime of the excited state and lies in the range of $10-50 \mathrm{MHz}$ for many well-studied host-guest systems [13-19]. Because of its sharpness, the ZPL is extremely sensitive to the molecule's local environment. The frequency of the ZPL can be shifted by mechanical strains or electric fields, including those caused by local degrees of freedom still active at the cryogenic temperature of the experiment. Such dynamics lead to spectral diffusion or to spectral jumps of the ZPL [20-22], and should therefore be eliminated or minimized for sensing applications. For example, the ZPL is very sensitive to librations of any methyl groups present in the host matrix [23]. Static hydrostatic pressure is also a well-known factor which shifts the ZPLs of single molecules [24-27]. The present work also relates to an earlier discovery by our group of acoustic modes localized at defects of the anthracene crystal [28]. Here, the tuning fork may be regarded as a well-known and well-controlled replacement for one of these localized modes. As we will see, its effects on the single-molecule lines are very comparable to those of the localized defect oscillators described previously.

The sensitivity of a molecular ZPL to the environment has its origin in the short-range interactions between the guest molecule and its first shell of host neighbors. Any variation of the relative positions of the guest and the host molecules induces a shift of the ZPL. Acoustic waves generated by a mechanical oscillator in contact with the sample will couple to the molecular transition and shift the ZPL of the guest molecule. We can thus use a single molecule to detect the local vibrations by monitoring the instantaneous frequency of its ZPL. In the present work, we chose single dibenzoterrylene (DBT) molecules embedded in an anthracene (Ac) crystal because of the stability of their ZPL, of their lifetime-limited linewidth and of their convenient wavelength [16].

Experimental.-The Ac single crystal doped with DBT molecules was glued to a quartz crystal tuning fork with a well-defined resonant oscillation frequency and high quality factor ( $Q$ factor), as shown in Fig. 1(a). By electrically 

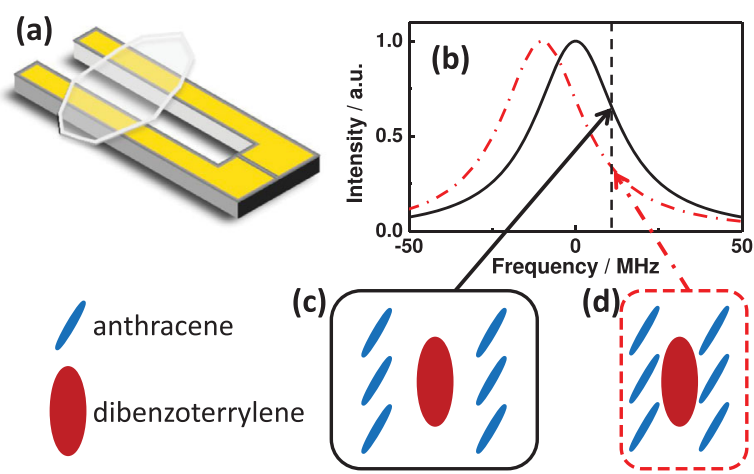

FIG. 1 (color). (a) Sample mounting: an anthracene crystal doped with DBT was attached to the quartz crystal tuning fork. (b) The ZPL of a single DBT molecule is shifted upon deformation of its surrounding host crystal, as shown in (c) and (d). The real deformations are three-dimensional and much more complicated, as molecules can also rotate and be distorted.

driving the tuning fork, the crystal is stretched or compressed periodically at the driving frequency. Such periodic vibrations change the average distance between molecules inside the crystal. The ZPLs of DBT molecules thus shift correspondingly, as shown in the cartoon of Fig. 1. Of course, the deformations of the molecular surroundings are much more complicated in reality and may involve bending, shearing, and molecular distortions. Any of these deformations, however, will give rise to a periodic shift of the molecular ZPL at the wave's frequency. When the excitation laser is tuned to the wing of the ZPL as indicated by the dashed vertical line in Fig. 1(b), a shift of the ZPL changes the molecular absorption and, thereby, the measured fluorescence intensity. In this way, the molecule's fluorescence can be used as a probe to read out the vibration amplitude, phase and frequency of the tuning fork, by monitoring time-dependent changes in the fluorescence intensity.

First, we characterized the tuning fork optically with the setup shown in Sec. 2(b) in the Supplemental Material $[29,30]$. The excitation laser was focused on the edge of one of the tuning fork prongs. The reflected light was collected and detected by a photodiode, providing a signal modulated at the oscillating frequency of the tuning fork. The lock-in signal thus provides the modulation amplitude of the reflected light at the oscillation frequency of the tuning fork. For the bare tuning fork in vacuum at $1.5 \mathrm{~K}$, a sharp peak was obtained at a frequency of $32.709 \mathrm{kHz}$ with a high $Q$ factor of about 40000 [Fig. 2(d)], corresponding to the fundamental flexion mode (symmetrical oscillation of the two prongs) [31]. When the sample crystal was attached, the oscillation of the tuning fork was strongly shifted and damped. Depending on the details of the contact between the crystal and the fork, the frequency shift could be positive or negative. The $Q$ factor was always lowered by the crystal. Surprisingly, three peaks rather than a single

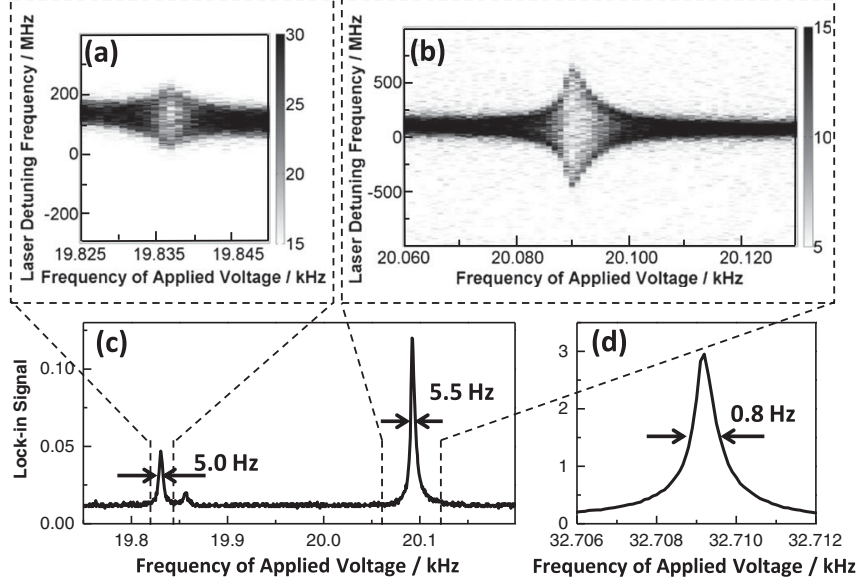

FIG. 2. Spectral trails of a single DBT molecule as functions of the driving frequency (a) from 19.820 to $19.850 \mathrm{kHz}$ or (b) from 20.060 to $20.130 \mathrm{kHz}$. The driving voltage was $0.8 \mathrm{~V}$. Lock-in signal of the reflection light intensity (c) from the tuning fork with sample attached and (d) from the bare tuning fork. The asymmetry of the main resonance around $20.091 \mathrm{kHz}$ is due to the anharmonicity of the tuning fork's oscillation. All the measurements were done in vacuum at $1.5 \mathrm{~K}$.

peak were observed in the range from 10 to $50 \mathrm{kHz}$, as shown in Fig. 2(c). Certain crystal defects can present acoustic modes at these frequencies, as was reported in Ref. [28]. However, our present experiments directly monitor the oscillation of the tuning fork and are therefore less sensitive to localized crystal modes. In addition, we did not observe any significant temperature dependence for these additional modes. We thus assign them to additional deformations of the tuning fork-crystal system, in addition to the strongest resonant peak located at $20.091 \mathrm{kHz}$ which we attribute to the damped fundamental flexion mode [32]. Theoretical calculation and modeling would be needed for a better understanding of the additional modes.

Results and discussion.- Spectral trails of a single DBT molecule were obtained by repeatedly scanning the laser frequency $(2 \mathrm{GHz}, 3 \mathrm{~s} / \mathrm{scan})$ while slowly varying the driving frequency on the tuning fork (19.820 to $19.850 \mathrm{kHz}$ and 20.060 to $20.130 \mathrm{kHz}$ ). Because of the limited fluorescence rate and time resolution, we could not directly monitor the oscillation of the ZPL shift. Instead, the periodical shift of the ZPL was observed as a broadening of the molecular line. As shown in Figs. 2(a) and 2(b), the broadening of the molecular ZPL resonated at the specific frequencies found by monitoring the displacement of the tuning fork [Fig. 2(c)]. These results are evidence for the coupling between the DBT molecule and the vibrations generated by the tuning fork in the Ac crystal. The slight asymmetry of the main peak is observed both on the lock-in signal from the tuning fork [Fig. 2(c)] and on the molecular spectral trail [Fig. 2(b)]. It is due to a frequency shift at high oscillation amplitudes and indicates anharmonicity of the tuning fork's oscillations. We studied the distortions of the 

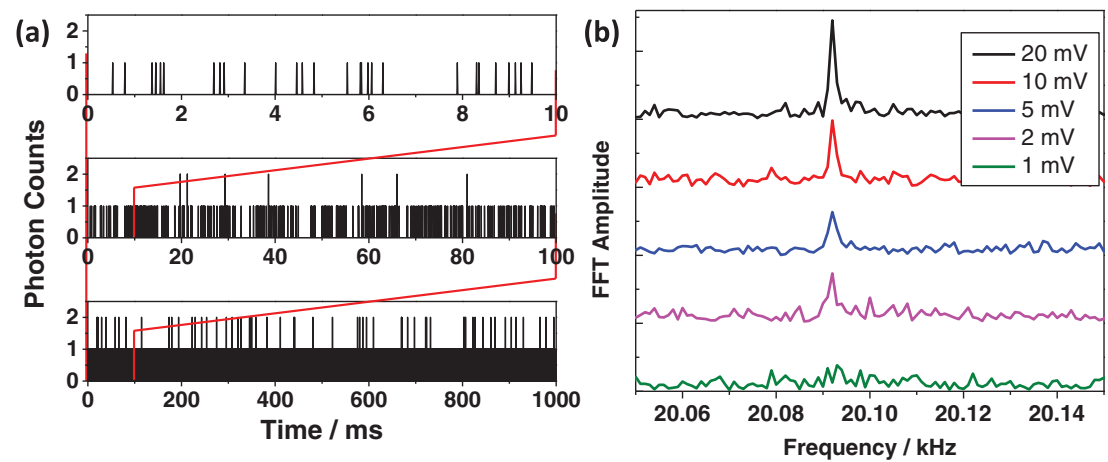

FIG. 3 (color). (a) Fluorescence photon counts (with bin size of $10 \mu$ s for periods of $10 \mathrm{~ms}, 100 \mathrm{~ms}$, and $1 \mathrm{~s}$ ) from a single DBT molecule (fluorescence rate of 3300 counts/s) and (b) fast Fourier transforms of such fluorescence intensity traces (1 s duration) when the tuning fork was driven at its resonant frequency $(20.091 \mathrm{kHz})$, for various driving voltages ( 1 to $20 \mathrm{mV}$ ). The curves are shifted along $Y$ axis for clarity. The sharp peak at $20.091 \mathrm{kHz}$ indicates the modulation due to coupling to the tuning fork. This signal becomes comparable to noise at a driving voltage of $1 \mathrm{mV}$.

resonance line with the oscillation amplitude, as shown in Sec. 4 in the Supplemental Material [29] and found that this effect is much enhanced by the crystal. Note that the resonance frequency there is different from Fig. 2(c) because the resonance frequency depends strongly on the attached crystal, which will be different for each measurement [32]. Similar observations were made on the localized acoustic defect modes [28].

To estimate the detection sensitivity of a single molecule, we fixed the laser frequency to the half maximum position of the ZPL [as shown by the dashed vertical line in Fig. 1(b)] and monitored the fluorescence photons from the molecule by time-tagged single-photon counting when the tuning fork was driven at resonant frequency $(20.091 \mathrm{kHz})$. The counted photons with bin size of $10 \mu \mathrm{s}$ over $10 \mathrm{~ms}, 100 \mathrm{~ms}$ and $1 \mathrm{~s}$ are shown in Fig. 3(a). Because of the low fluorescence signal, we cannot directly see the modulation of the fluorescence intensity trace, not even for the largest oscillation amplitude. However, a fast Fourier transform (FFT) of the fluorescence intensity trace reveals the weak modulation, as shown in Fig. 3(b) for different oscillation amplitudes. Note that, in spite of the random distribution of single photon detection events, the FFT picks up the weak modulated component unambiguously, just as a lock-in detection would filter an analog signal. The sharp peaks represent the driving frequency (their linewidth is determined by the total integration time). The FFT signal amplitude varies linearly with the driving voltage, i.e., with the tuning fork's oscillation amplitude. As a consequence, the FFT signal can no longer be distinguished from noise, for about $1 \mathrm{mV}$ driving voltage with an integration time of $1 \mathrm{~s}$.

Hereafter, we discuss the detection sensitivity following the reasoning and notations of Puller et al. [11]. We measure the oscillator's displacement in relative deformation of the host lattice $\varepsilon=\delta x / x$, rather than the absolute displacement of the nanotube's tip as done in Ref. [11]. The sensitivity is determined by the smallest deformation detectable over a time $t$ against shot noise fluctuations of the fluorescence signal, $\sqrt{I t}$, where $I$ is the fluorescence rate. The shift of the molecular frequency is given by $\delta \nu=\varepsilon(d \nu / d \varepsilon)$. It gives rise to an intensity change $\delta I=I \delta \nu / \Gamma$, where $\Gamma$ is the linewidth $(30 \mathrm{MHz}$ in previous experiments, $80 \mathrm{MHz}$ in our case). The minimum deformation value at which the signal overcomes the photon noise is thus $\varepsilon_{m}=\Gamma(d \varepsilon / d \nu) \sqrt{(t / I)}$. The elastic modulus of anthracene crystal is $E \sim 10^{10} \mathrm{~N} / \mathrm{m}^{2}$ corresponding to $10^{5}$ bar $[33,34]$. The frequency shift induced by pressure change is reported to be $\sim 1 \mathrm{GHz} /$ bar [24]; i.e., a $1 \mathrm{GHz}$ shift corresponds to a lattice deformation $\varepsilon=10^{-5}$. The coupling constant, corresponding to a change of frequency of the molecular transition per relative deformation of the crystal lattice is thus about $d \nu / d \varepsilon=10^{14} \mathrm{~Hz}$. Therefore, the typical values in single-molecule spectroscopy give $\varepsilon_{m}=10^{-9} \sqrt{t}$, i.e., a deformation of $1 \mathrm{ppb} / \sqrt{\mathrm{Hz}}$. With a low fluorescent rate of 3300 counts/s and a broad linewidth in our experiment, we would expect a sensitivity of about $1.4 \times 10^{-8} \mathrm{~Hz}^{-1 / 2}$. However, the experimental detection limit is rather $7 \times 10^{-7} \mathrm{~Hz}^{-1 / 2}$ (See calculation in Sec. 3 in the Supplemental Material [29]). We attribute this difference of a factor 50 to the background mechanical vibrations generated by the noisy environment of our cryostat. Indeed, a noise background appears in the FFT of the intensity traces, as shown in Sec. 5 in the Supplemental Material [29]. This noise background was also observed in the reflection measurement where no molecular spectroscopy was involved. Therefore, the oscillations of the tuning fork are driven by noise sources. We estimated the energy of these background oscillations to around $10^{-17} \mathrm{~J}$ (see Sec. 5 in the Supplemental Material [29]), and found them to be much larger than thermal fluctuations $\left(10^{-23} \mathrm{~J}\right)$ expected at our experimental temperature $(1.5 \mathrm{~K})$. These vibrations ought to be eliminated to reach the shot-noise limited sensitivity of our singlemolecule acoustic detector.

We now discuss the theoretical detection limits for various oscillators. For the vibration amplitude of the 
tuning fork's prongs, which linearly depends on the driving voltage [35-37], we can estimate the experimental sensitivity of the fork prong's displacement to about $0.14 \mathrm{~nm} / \sqrt{\mathrm{Hz}}$ (see more details in Sec. 3 in the Supplemental Material [29]). If the shot-noise-limited sensitivity is achieved, much weaker displacements still are detectable with the molecule. Assuming the oscillation to be a mode delocalized over the whole crystal, typically $100 \mu \mathrm{m}$ in size (as in the present and previous work [28]), the detectable displacement is about $1.4 \mathrm{pm} / \sqrt{\mathrm{Hz}}$ in our present conditions, and would reach $100 \mathrm{fm} / \sqrt{\mathrm{Hz}}$ with improvements in the fluorescence collection efficiency. A nano-oscillator with $10 \mathrm{~nm}$ in size located around the molecule would give a sensitivity of $10 \mathrm{am} / \sqrt{\mathrm{Hz}}$. This sensitivity exceeds that of the device proposed by Puller et al. based on an oscillating carbon nanotube placed in the vicinity of the molecule [11]. Another important feature of our acoustic detector is its time resolution. The frequency of nanomechanical oscillators covers a large range from $\mathrm{MHz}$ to $\mathrm{THz}[38,39]$. The time resolution of our current setup is limited to $25 \mathrm{MHz}$ by the dead time of our photodetector. Even with a faster photodetector, the time resolution will be limited to some hundreds of $\mathrm{MHz}$ by the fluorescence lifetime of the molecules. Higher bandwidths would require faster emitters, for example, molecules coupled to plasmonic antennas.

As a final remark, we rule out the possibility of a Stark shift of the molecular transition due to the applied electric field for driving the tuning fork [40,41]. Indeed, Fig. 2 shows that the resonant acoustic effect is much larger than the frequency-independent Stark effect. Moreover, we can estimate the electric field on the molecule to $5 \mathrm{kV} / \mathrm{m}$ for an applied voltage of $0.8 \mathrm{~V}$ on the tuning fork. Such a weak field cannot induce a significant Stark effect on the molecule $[28,41]$. The observed ZPL shift of the DBT molecule is therefore exclusively due to the mechanical coupling between the molecule and the tuning fork.

Conclusion.-In conclusion, we measured the coupling of a single organic molecule to acoustic strain generated by a macroscopic mechanical oscillator. Exciting the fluorescence at half-maximum gave rise to an intensity modulation of the fluorescence intensity trace. Such a weak modulation was successfully detected through a fast Fourier transform that works as a lock-in amplifier for a digital signal. The sensitivity threshold to relative deformation reached in our current experiments was about $7 \times 10^{-7} \mathrm{~Hz}^{-1 / 2}$, limited by mechanical experimental noise. However, for a shot-noiselimited detection of the vibrations of a nanomechanical oscillator, the sensitivity should reach the level of $1 \mathrm{fm} / \sqrt{\mathrm{Hz}}$, comparable to the theoretical sensitivity proposed by Puller et al. [11]. Such a high sensitivity is promising for reading out the quantum states of nanomechanical devices. Even though the detection sensitivity is not exceptionally high compared to other techniques, the main advantage of single-molecule detection is the small size of the sensor (subnanometer in size). These small probes can be placed in the elastic strain field of the oscillator, enabling high coupling to artificial nanomechanical oscillators [11], or to natural oscillators found around defects in crystals [28] and in disordered solids [42].

This work is supported by the Stichting voor Fundamenteel Onderzoek der Materie (FOM), which is part of the Dutch Science Funding Organization NWO.

* Corresponding author.

orrit@physics.leidenuniv.nl

†Present address: Chemical Physics, Lund University, Box 124, 22100 Lund, Sweden.

[1] F. Dunn and W. J. Fry, J. Acoust. Soc. Am. 31, 632 (1959).

[2] R. G. Maev, Acoustic Microscopy (Wiley-VCH Verlag GmbH \& Co. KGaA, Weinheim, Germany, 2008).

[3] J. Gieseler, B. Deutsch, R. Quidant, and L. Novotny, Phys. Rev. Lett. 109, 103603 (2012).

[4] A. Ohlinger, A. Deak, A. A. Lutich, and J. Feldmann, Phys. Rev. Lett. 108, 018101 (2012).

[5] E. Gil-Santos, D. Ramos, J. Martínez, M. FernándezRegúlez, R. García, A. San Paulo, M. Calleja, and J. Tamayo, Nat. Nanotechnol. 5, 641 (2010).

[6] G. Longo, L. Alonso-Sarduy, L. M. Rio, A. Bizzini, A. Trampuz, J. Notz, G. Dietler, and S. Kasas, Nat. Nanotechnol. 8, 522 (2013).

[7] N. E. Flowers-Jacobs, D. R. Schmidt, and K. W. Lehnert, Phys. Rev. Lett. 98, 096804 (2007).

[8] S. Etaki, M. Poot, I. Mahboob, K. Onomitsu, H. Yamaguchi, and H. S. J. van der Zant, Nat. Phys. 4, 785 (2008).

[9] R. G. Knobel and A. N. Cleland, Nature (London) 424, 291 (2003).

[10] M. D. LaHaye, J. Suh, P. M. Echternach, K. C. Schwab, and M. L. Roukes, Nature (London) 459, 960 (2009).

[11] V. Puller, B. Lounis, and F. Pistolesi, Phys. Rev. Lett. 110, 125501 (2013).

[12] W. E. Moerner and M. Orrit, Science 283, 1670 (1999).

[13] M. Orrit and J. Bernard, Phys. Rev. Lett. 65, 2716 (1990).

[14] A.-M. Boiron, F. Jelezko, Y. Durand, B. Lounis, and M. Orrit, Mol. Cryst. Liq. Cryst. 291, 41 (1996).

[15] F. Jelezko, P. Tamarat, B. Lounis, and M. Orrit, J. Phys. Chem. 100, 13892 (1996).

[16] A. A. L. Nicolet, C. Hofmann, M. A. Kol'chenko, B. Kozankiewicz, and M. Orrit, ChemPhysChem 8, 1215 (2007).

[17] A. A. Gorshelev, A. V. Naumov, I. Y. Eremchev, Y. G. Vainer, L. Kador, and J. Köhler, ChemPhysChem 11, 182 (2010).

[18] A. Walser, A. Renn, S. Götzinger, and V. Sandoghdar, Chem. Phys. Lett. 472, 44 (2009).

[19] P. Navarro, Y. Tian, M. van Stee, and M. Orrit, ChemPhysChem, doi:10.1002/cphc.201402200 (2014).

[20] W. P. Ambrose and W. E. Moerner, Nature (London) 349, 225 (1991).

[21] T. Basché, S. Kummer, and C. Bräuchle, Nature (London) 373, 132 (1995).

[22] F. Kulzer, S. Kummer, R. Matzke, C. Brauchle, and T. Basché, Nature (London) 387, 688 (1997). 
[23] Y. Tian, P. Navarro, B. Kozankiewicz, and M. Orrit, ChemPhysChem 13, 3510 (2012).

[24] A. A. L. Nicolet, P. Bordat, C. Hofmann, M. A. Kol'chenko, B. Kozankiewicz, R. Brown, and M. Orrit, ChemPhysChem 8, 1929 (2007).

[25] M. Croci, H.-J. Müschenborn, F. Güttler, A. Renn, and U. P. Wild, Chem. Phys. Lett. 212, 71 (1993).

[26] T. Iwamoto, A. Kurita, and T. Kushida, Chem. Phys. Lett. 284, 147 (1998).

[27] A. Müller, W. Richter, and L. Kador, Chem. Phys. Lett. 241, 547 (1995).

[28] M. A. Kol'chenko, A. A. L. Nicolet, M. D. Galouzis, C. Hofmann, B. Kozankiewicz, and M. Orrit, New J. Phys. 11, 023037 (2009).

[29] See the Supplemental Material at http://link.aps.org/ supplemental/10.1103/PhysRevLett.113.135505, which includes Ref. [30], for experimental, amplitude of the bare tuning fork, estimation of the amplitude of the tuning fork with attached Ac crystal, anharmonic effect, noise background, and autocorrelation function of the fluorescence time trace.

[30] D. van Vörden, M. Lange, M. Schmuck, N. Schmidt, and R. Möller, Beilstein J. Nanotechnol. 3, 809 (2012).

[31] J.-M. Friedt and E. Carry, Am. J. Phys. 75, 415 (2007).
[32] The resonant frequency of the tuning fork with attached crystal varies from time to time for different Ac crystals and different cooling cycles.

[33] R. C. Dye and C. J. Eckhardt, J. Chem. Phys. 90, 2090 (1989).

[34] H. B. Huntington, S. G. Gangoli, and J. L. Mills, J. Chem. Phys. 50, 3844 (1969).

[35] A. Castellanos-Gomez, C. R. Arroyo, N. Agraït, and G. Rubio-Bollinger, Microsc. Microanal. 18, 353 (2012).

[36] P. Sandoz, J.-M. Friedt, and E. Carry, Rev. Sci. Instrum. 79, 086102 (2008).

[37] A. G. Ruiter, K. O. van der Werf, J. A. Veerman, M. F. Garcia-Parajo, W. H. Rensen, and N. F. van Hulst, Ultramicroscopy 71, 149 (1998).

[38] G. Anetsberger, O. Arcizet, Q.P. Unterreithmeier, R. Riviére, A. Schliesser, E. M. Weig, J. P. Kotthaus, and T. J. Kippenberg, Nat. Phys. 5, 909 (2009).

[39] V. V. Temnov, Nat. Photonics 6, 728 (2012).

[40] M. Orrit, J. Bernard, A. Zumbusch, and R. I. Personov, Chem. Phys. Lett. 196, 595 (1992).

[41] C. Brunel, P. Tamarat, B. Lounis, J. C. Woehl, and M. Orrit, J. Phys. Chem. A 103, 2429 (1999).

[42] Y. G. Vainer, A. V. Naumov, M. Bauer, and L. Kador, Phys. Rev. Lett. 97, 185501 (2006). 